\title{
Examining the Geometrical Model with Inverted Mass Hierarchy for Neutrinos
}

\author{
Mizue Honda ${ }^{1}$ and Morimitsu Tanimoto ${ }^{2}$ \\ ${ }^{1}$ Graduate School of Science and Technology, Niigata University, Niigata 950-2181, Japan \\ ${ }^{2}$ Department of Physics, Niigata University, Niigata 950-2181, Japan
}

\begin{abstract}
The comprehensive analyses are presented in the model with the inverted mass hierarchy for neutrinos, which follows from a geometrical structure of a $(1+5)$ dimensional space-time where two extra dimensions are compactified on the $\mathbf{T}^{\mathbf{2}} / \mathbf{Z}_{\mathbf{3}}$ orbifold. The model gives two large lepton flavor mixings due to the $S_{3}$ structure in the $(1+5)$ dimensional space-time. It also predicts the lightest neutrino mass as $m_{3}=(1-50) \times 10^{-5}$ $\mathrm{eV}$ and the effective neutrino mass responsible for neutrinoless double beta decays as $\langle m\rangle_{e e} \simeq 50 \mathrm{meV}$. The low energy $C P$ violation, $J_{C P}$ could be 0.02 . On the other hand, the observed baryon asymmetry in the present universe is produced by the non-thermal leptogenesis, which works even at the reheating temperature $T_{R}=10^{4}-10^{6} \mathrm{GeV}$. The correlation between the baryon asymmetry and the low energy $C P$ violation is examined in this model.
\end{abstract}




\section{Introduction}

It is the important task to find an origin of the observed hierarchies in masses and flavor mixings for quarks and leptons. In particular, the geometrical aspect provides a progressive study for masses and mixings of quarks and leptons. The non-Abelian discrete flavor symmetry is also realized in the simple geometrical understanding of superstring theory [1]. Recently, a higher-dimensional model of neutrinos [2] was proposed based on the orbifold model [3]. In this model, extra two dimensions in a $(1+5)$ dimensional space-time are compactified on the $\mathbf{T}^{\mathbf{2}} / \mathbf{Z}_{\mathbf{3}}$ orbifold, which has three equivalent fixed points. The quarks and leptons are supposed to belong to $5^{*}$ and $\mathbf{1 0}$ in the $\mathrm{SU}(5)$ grand unification model. A $5^{*}$ and a right-handed neutrino $N$ in each family are localized on each of the three fixed points of the orbifold, while three 10's live in the bulk. Assuming the discrete flavor symmetry $S_{3}$, the successful democratic mass matrices for quarks and leptons are obtained, provided that the three 10's and Higgs doublets are distributed homogeneously in the bulk. On the other hand, the Higgs $\phi$ for the B-L breaking is localized on one of the three fixed points. Therefore, one of $N$ acquires a superheavy Majorana mass generating an inverted hierarchy in the neutrino mass spectrum.

The previous work [2] has shown that the model is consistent with observations on the neutrino masses and mixings. In particular, the leptogenesis works well with the reheating temperature $T_{R}=10^{7}-10^{10} \mathrm{GeV}$. However, a CP violating phase is only taken account for simplicity in the previous work. In this paper, we present comprehensive numerical analyses including all possible $\mathrm{CP}$ violating phases to test the model in future

experiments. Moreover, we scan wider regions of parameters to be consistent with all data of the neutrino masses and mixings. We have found that the leptogenesis works at the lower reheating temperature $T_{R} \simeq 10^{4}-10^{6} \mathrm{GeV}$.

In section 2, we summarize the model for the neutrino and charged lepton mass matrices, where the inverted neutrino mass spectrum is naturally obtained. In section 3, 
numerical analyses are given for the neutrino masses and mixings. In section 4 , the nonthermal leptogenesis is numerically studied. The correlation between the leptogenesis and the low energy $C P$ violation is also examined. The summary is devoted to section 5 .

\section{Framework of the Model}

Let us begin with presenting the model [2], which is based on works in [3, 4]. As shown in Fig. 1, a $5_{\mathbf{i}}{ }^{*}$ and a right-handed neutrino $N_{i}$ in each family are localized on one of the equivalent three fixed points of the $\mathbf{T}^{\mathbf{2}} / \mathbf{Z}_{\mathbf{3}}$ orbifold while three $\mathbf{1 0}_{\mathbf{i}}$ 's and Higgs doublets $H_{u}$ and $H_{d}$ live in the bulk. The $\phi$ for the B-L breaking is localized on the fixed point on which the $\mathbf{5}_{3}^{*}$ and $N_{3}$ in the third family reside. Therefore, the $N_{3}$ in the third family has a very large Majorana mass compared with those of other N's.

Figure 1: The configuration of matters and Higgses at three fixed

Since the three fixed points are equivalent to one

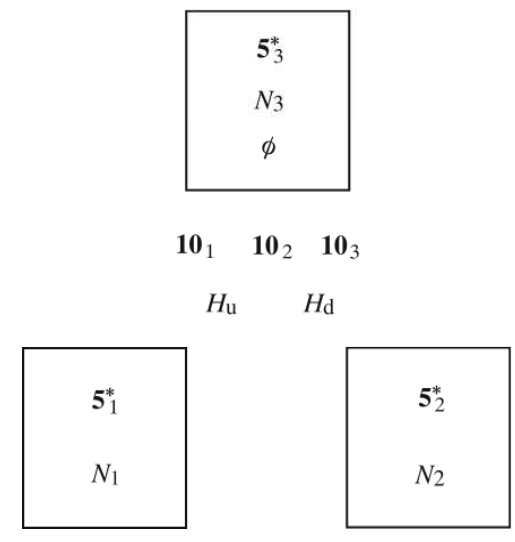
points and in the bulk.

another, we assume an $S_{3}$ family symmetry acting on three $5^{*}$ 's and on three $N$ 's. We also introduce breakings of the $S_{3}$ symmetry to obtain realistic mass matrices. We assume two sources of the breaking. One is a localization of the wave function of the $\phi$ field and the other is small distortions of the wave functions of the three 10's and the doublet Higgses from homogeneous forms in the bulk.

\subsection{Neutrino Mass Matrix}

The Dirac neutrino mass matrix is given by the Yukawa coupling matrix of $N 5^{*} H_{u}$. There are two independent matrices which are invariant of the $S_{3}$ symmetry [5, 6]. The 
$S_{3}$ invariant Dirac mass matrix is given by [5]

$$
h_{\nu}=h_{0}\left[\left(\begin{array}{lll}
1 & 0 & 0 \\
0 & 1 & 0 \\
0 & 0 & 1
\end{array}\right)+\left(\begin{array}{ccc}
0 & \bar{\epsilon} & \bar{\epsilon} \\
\bar{\epsilon} & 0 & \bar{\epsilon} \\
\bar{\epsilon} & \bar{\epsilon} & 0
\end{array}\right)\right] \text {. }
$$

The parameter $\bar{\epsilon}$ is suppressed by separation of the three fixed points [7].

We have assumed a homogeneous distribution of the Higgs fields $H_{u}$ in the bulk. However, a small distortion of the Higgs wave function may induce a violation of the $S_{3}$ symmetry. The breaking effects appear first in the diagonal elements of the above mass matrix and the effects in the $\bar{\epsilon}$ term may be negligible for our discussion. Therefore, the neutrino Dirac mass matrix is given by

$$
h_{\nu}=h_{0}\left[\left(\begin{array}{ccc}
1 & 0 & 0 \\
0 & 1+\bar{\delta}_{1} & 0 \\
0 & 0 & 1+\bar{\delta}_{2}
\end{array}\right)+\left(\begin{array}{ccc}
0 & \bar{\epsilon} & \bar{\epsilon} \\
\bar{\epsilon} & 0 & \bar{\epsilon} \\
\bar{\epsilon} & \bar{\epsilon} & 0
\end{array}\right)\right]=h_{0}\left(\begin{array}{ccc}
1 & \bar{\epsilon} & \bar{\epsilon} \\
\bar{\epsilon} & 1+\bar{\delta}_{1} & \bar{\epsilon} \\
\bar{\epsilon} & \bar{\epsilon} & 1+\bar{\delta}_{2}
\end{array}\right) .
$$

Here, $\bar{\delta}_{1}, \bar{\delta}_{2}$ and $\bar{\epsilon}$ are parametrized as

$$
\bar{\delta}_{1}=\delta_{1} e^{i \beta_{1}}, \quad \bar{\delta}_{2}=\delta_{2} e^{i \beta_{2}}, \quad \bar{\epsilon}=\epsilon e^{i \varphi}
$$

where $\delta_{1}, \delta_{2}$ and $\epsilon$ are real parameters, and $\beta_{1}, \beta_{2}$ and $\varphi$ are CP violating phases.

The Majorana mass matrix $M_{R}$ for the right-handed neutrino $N_{i}(i=1,2,3)$ is determined also by localization properties of $N_{i}$ and $\phi$ fields. Since the $\phi$ is assumed to reside on one of the three fixed points where the third family $N_{3}$ is localized, the $(3,3)$ element of the Majorana mass matrix dominates over other elements. Then, the Majorana mass matrix is given as follows:

$$
M_{R}=M_{0}\left(\begin{array}{ccc}
\bar{\epsilon}_{2} & \bar{\epsilon}_{3}^{2} & \bar{\epsilon}_{1} \\
\bar{\epsilon}_{3}^{2} & \bar{\epsilon}_{2} & \bar{\epsilon}_{1} \\
\bar{\epsilon}_{1} & \bar{\epsilon}_{1} & 1
\end{array}\right)
$$

where $\bar{\epsilon}_{1}, \bar{\epsilon}_{2}$ and $\bar{\epsilon}_{3}$ are three independent suppression factors. These are parametrized as

$$
\bar{\epsilon}_{1}=\epsilon_{1} e^{i \alpha_{1}}, \quad \bar{\epsilon}_{2}=\epsilon_{2} e^{i \alpha_{2}}, \quad \bar{\epsilon}_{3}=\epsilon_{3} e^{i \alpha_{3}}
$$

where $\epsilon_{1}, \epsilon_{2}$ and $\epsilon_{3}$ are real parameters, and $\alpha_{1}, \alpha_{2}$ and $\alpha_{3}$ are CP violating phases. Notice that the suppression factors come from the separation of the distinct fixed points and hence the parameters $\epsilon_{i}(i=1,2,3)$ are the same order of the magnitude of $\epsilon$. 
Through the seesaw mechanism [8, 9], the effective neutrino mass matrix is given, in the leading order of $\bar{\epsilon}, \bar{\epsilon}_{i}$ and $\bar{\delta}_{i}$, by

$$
M_{\nu}=h_{\nu}^{T} M_{R}^{-1} h_{\nu} \simeq \frac{h_{0}^{2}}{\bar{\epsilon}_{2} M_{0}}\left(\begin{array}{ccc}
1-\bar{\epsilon}_{1}^{2} & 2 \bar{\epsilon}+\tilde{\epsilon} & \bar{\epsilon}-\bar{\epsilon}_{1} \\
2 \bar{\epsilon}+\tilde{\epsilon} & 1+2 \bar{\delta}_{1}-\bar{\epsilon}_{1}^{2} & \bar{\epsilon}-\bar{\epsilon}_{1} \\
\bar{\epsilon}-\bar{\epsilon}_{1} & \bar{\epsilon}-\bar{\epsilon}_{1} & \bar{\epsilon}_{2}
\end{array}\right)
$$

where

$$
\tilde{\epsilon}=\frac{\bar{\epsilon}_{1}^{2}-\bar{\epsilon}_{3}^{2}}{\bar{\epsilon}_{2}}
$$

Since the neutrino mass matrix is complex, we discuss $M_{\nu} M_{\nu}^{\dagger}$ :

$$
M_{\nu} M_{\nu}^{\dagger} \simeq \frac{h_{0}^{4}}{\epsilon_{2}^{2} M_{0}^{2}}\left(\begin{array}{ccc}
1-\frac{2 \epsilon_{1}^{2} \cos \left(2 \alpha_{1}-\alpha_{2}\right)}{\epsilon_{2}} & 4 \epsilon \cos \varphi+2 \tilde{\epsilon}^{\prime} & \bar{\epsilon}^{*}-\bar{\epsilon}_{1}^{*} \\
4 \epsilon \cos \varphi+2 \tilde{\epsilon}^{\prime} & 1-\frac{2 \epsilon_{1}^{2} \cos \left(2 \alpha_{1}-\alpha_{2}\right)}{\epsilon_{2}}+4 \delta_{1} & \bar{\epsilon}^{*}-\bar{\epsilon}_{1}^{*} \\
\bar{\epsilon}-\bar{\epsilon}_{1} & \bar{\epsilon}-\bar{\epsilon}_{1} & \epsilon_{2}^{2}
\end{array}\right)
$$

with

$$
\tilde{\epsilon}^{\prime}=\frac{\epsilon_{1}^{2} \cos \left(2 \alpha_{1}-\alpha_{2}\right)-\epsilon_{3}^{2} \cos \left(2 \alpha_{3}-\alpha_{2}\right)}{\epsilon_{2}} .
$$

Then, the squares of the neutrino mass eigenvalues are given as

$$
m_{1}^{2} \simeq \frac{h_{0}^{4}}{\epsilon_{2}^{2} M_{0}^{2}}, \quad m_{2}^{2} \simeq \frac{h_{0}^{4}}{\epsilon_{2}^{2} M_{0}^{2}}\left(1-4 \tilde{\epsilon}^{\prime}+4 \delta_{1}\right), \quad m_{3}^{2} \simeq \frac{h_{0}^{4}}{\epsilon_{2}^{2} M_{0}^{2}} \epsilon_{2}^{2},
$$

which are the spectrum called as the inverted mass hierarchy. Therefore, the ratio of the solar neutrino mass scale and the atmospheric neutrino mass scale is given as

$$
\frac{\Delta m_{\mathrm{solar}}^{2}}{\Delta m_{\mathrm{atm}}^{2}}=\frac{m_{2}^{2}-m_{1}^{2}}{m_{1}^{2}-m_{3}^{2}} \simeq 4\left(\delta_{1}-\tilde{\epsilon}^{\prime}\right) .
$$

The experimental data of $\Delta m_{\text {solar }}^{2}$ and $\Delta m_{\text {atm }}^{2}$ [10, 11, 12, give us an allowed region in the parameter space.

The unitary matrix, which diagonalizes the neutrino mass matrix of Eq. ([6) such as $V_{\nu}^{T} M_{\nu} V_{\nu}=M_{\text {diagonal }}$, is given approximately by

$$
V_{\nu} \simeq\left(\begin{array}{ccc}
1 & \frac{2 \epsilon \cos \varphi+\tilde{\epsilon}^{\prime \prime}}{2 \delta_{1}} & \mathcal{O}\left(\epsilon, \epsilon_{i}\right) \\
-\frac{2 \epsilon \cos \varphi+\tilde{\epsilon}^{\prime \prime}}{2 \delta_{1}} & 1 & \mathcal{O}\left(\epsilon, \epsilon_{i}\right) \\
\mathcal{O}\left(\epsilon, \epsilon_{i}\right) & \mathcal{O}\left(\epsilon, \epsilon_{i}\right) & 1
\end{array}\right)
$$

with

$$
\tilde{\epsilon}^{\prime \prime}=\frac{2 \epsilon_{1}^{2} \cos \left(2 \alpha_{1}-\alpha_{2}\right)-\epsilon_{3}^{2} \cos \left(2 \alpha_{3}-\alpha_{2}\right)}{\epsilon_{2}}
$$

where $C P$ violating phases appear in $(1,3),(3,1),(2,3)$ and $(3,2)$ elements. 


\subsection{Charged Lepton Mass Matrix}

When the three 10's and the Higgs multiplet $H_{d}$ distribute homogeneously in the bulk, one obtains the democratic mass matrix for charged leptons. Now we introduce distortions of wave functions of the 10's in the bulk. We assume that dominant effects appear on the diagonal elements of the above matrix. Then, the charged lepton mass matrix is given by

$$
M_{\ell}=\frac{m_{0}}{3}\left(\begin{array}{lll}
1 & 1 & 1 \\
1 & 1 & 1 \\
1 & 1 & 1
\end{array}\right)+\left(\begin{array}{ccc}
\delta_{\ell 1} & 0 & 0 \\
0 & \delta_{\ell 2} & 0 \\
0 & 0 & \delta_{\ell 3}
\end{array}\right),
$$

where the second term of the right-hand side is the $S_{3}$ breaking terms coming from the distortions of the $\mathbf{1 0}$ fields. We take all $\delta_{\ell i}$ to be real, for simplicity. This form of the mass matrix is used in Ref. [13, 5]. Here, we have assumed that the effects of distortion of the Higgs field $H_{d}$ are negligibly small.

The matrix of Eq. (14) is diagonalized by $V_{\ell}=F_{0} L_{\ell}$, where

$$
\begin{aligned}
& F_{0}=\left(\begin{array}{ccc}
\frac{1}{\sqrt{2}} & \frac{1}{\sqrt{6}} & \frac{1}{\sqrt{3}} \\
-\frac{1}{\sqrt{2}} & \frac{1}{\sqrt{6}} & \frac{1}{\sqrt{3}} \\
0 & -\frac{2}{\sqrt{6}} & \frac{1}{\sqrt{3}}
\end{array}\right), \\
& F_{\ell} \simeq\left(\begin{array}{ccc}
\cos \theta_{\ell} & \sin \theta_{\ell} & \lambda_{\ell} \sin 2 \theta_{\ell} \\
-\sin \theta_{\ell} & \cos \theta_{\ell} & -\lambda_{\ell} \cos 2 \theta_{\ell} \\
-\lambda_{\ell} \sin 3 \theta_{\ell} & \lambda_{\ell} \cos 3 \theta_{\ell} & 1
\end{array}\right),
\end{aligned}
$$

with

$$
\begin{aligned}
& \tan 2 \theta_{\ell} \simeq \sqrt{3} \frac{\delta_{\ell 2}-\delta_{\ell 1}}{2 \delta_{\ell 3}-\delta_{\ell 2}-\delta_{\ell 1}}, \quad \lambda_{\ell} \simeq \frac{1}{3 \sqrt{2}} \frac{\xi_{\ell}}{m_{0}}, \\
& \xi_{\ell}=\sqrt{\left(2 \delta_{\ell 3}-\delta_{\ell 2}-\delta_{\ell 1}\right)^{2}+3\left(\delta_{\ell 2}-\delta_{\ell 1}\right)^{2}} .
\end{aligned}
$$

The mass eigenvalues are given by

$$
\begin{aligned}
m_{e} & =\frac{1}{3}\left(\delta_{\ell 1}+\delta_{\ell 2}+\delta_{\ell 3}\right)-\frac{1}{6} \xi_{\ell}, \\
m_{\mu} & =\frac{1}{3}\left(\delta_{\ell 1}+\delta_{\ell 2}+\delta_{\ell 3}\right)+\frac{1}{6} \xi_{\ell}, \\
m_{\tau} & =m_{0}+\frac{1}{3}\left(\delta_{\ell 1}+\delta_{\ell 2}+\delta_{\ell 3}\right) .
\end{aligned}
$$

If we take $\delta_{\ell 1}+\delta_{\ell 2}=0$ and $\delta_{\ell 2} \ll \delta_{\ell 3}$, for simplicity [5], we get following mixings in terms 
of the charged lepton masses:

$$
\sin \theta_{\ell} \simeq-\sqrt{\frac{m_{e}}{m_{\mu}}}, \quad \lambda_{\ell} \simeq \frac{1}{\sqrt{2}} \frac{m_{\mu}}{m_{\tau}}
$$

which are used in our numerical calculations. It may be important to note that the condition $\delta_{\ell 1}+\delta_{\ell 2}=0$ is crucial for the prediction of $\sin \theta_{\ell}$.

\subsection{Right-handed Majorana Neutrino Mass Matrix}

The right-handed Majorana neutrino mass matrix is examined to discuss the leptogenesis. Solving the hermitian matrix $M_{R} M_{R}^{\dagger}$,

$$
\begin{aligned}
& M_{R} M_{R}^{\dagger}=M_{0}^{2} \times \\
& \left(\begin{array}{ccc}
\epsilon_{1}^{2}+\epsilon_{2}^{2}+\epsilon_{3}^{4} & \epsilon_{1}^{2}+2 \epsilon_{2} \epsilon_{3}^{2} \cos \left(\alpha_{2}-2 \alpha_{3}\right) & \bar{\epsilon}_{1}+\bar{\epsilon}_{1}^{*} \bar{\epsilon}_{2}+\bar{\epsilon}_{1}^{*} \bar{\epsilon}_{3}^{2} \\
\epsilon_{1}^{2}+2 \epsilon_{2} \epsilon_{3}^{2} \cos \left(\alpha_{2}-2 \alpha_{3}\right) & \epsilon_{1}^{2}+\epsilon_{2}^{2}+\epsilon_{3}^{4} & \bar{\epsilon}_{1}+\bar{\epsilon}_{1}^{*} \bar{\epsilon}_{2}+\bar{\epsilon}_{1}^{*} \bar{\epsilon}_{3}^{2} \\
\bar{\epsilon}_{1}^{*}+\bar{\epsilon}_{1} \bar{\epsilon}_{2}^{*}+\bar{\epsilon}_{1} \bar{\epsilon}_{3}^{* 2} & \bar{\epsilon}_{1}^{*}+\bar{\epsilon}_{1} \bar{\epsilon}_{2}^{*}+\bar{\epsilon}_{1} \bar{\epsilon}_{3}^{* 2} & 2 \epsilon_{1}^{2}+1
\end{array}\right),
\end{aligned}
$$

we get the squares of mass eigenvalues as follows:

$$
\begin{aligned}
& m_{R 1}^{2} \simeq M_{0}^{2}\left[\epsilon_{2}^{2}-4 \epsilon_{1}^{2} \epsilon_{2} \cos \left(2 \alpha_{1}-\alpha_{2}\right)+2 \epsilon_{2} \epsilon_{3}^{2} \cos \left(\alpha_{2}-2 \alpha_{3}\right)\right] \\
& m_{R 2}^{2} \simeq M_{0}^{2}\left[\epsilon_{2}^{2}-2 \epsilon_{2} \epsilon_{3}^{2} \cos \left(\alpha_{2}-2 \alpha_{3}\right)\right] \\
& m_{R 3}^{2} \simeq M_{0}^{2}\left[1+4 \epsilon_{1}^{2} \cos 2 \alpha_{1}+4 \epsilon_{1}^{2} \epsilon_{2} \cos \left(2 \alpha_{1}-\alpha_{2}\right)\right]
\end{aligned}
$$

It is remarked that the right-handed Majorana neutrinos of the first and second family are almost degenerated. The unitary matrix to diagonalize $M_{R} M_{R}^{\dagger}$ is given by

$$
U_{R} \simeq\left(\begin{array}{ccc}
\frac{1}{\sqrt{2}} & \frac{1}{\sqrt{2}} & \bar{\epsilon}_{1} \\
\frac{1}{\sqrt{2}} & -\frac{1}{\sqrt{2}} & \bar{\epsilon}_{1} \\
-\sqrt{2} \bar{\epsilon}_{1}^{*} & 0 & 1
\end{array}\right) P
$$

where $P$ is the phase matrix, which is ignored in the following calculations because it does not affect our results.

In order to discuss the leptogenesis later, we take the diagonal basis of the right-handed Majorana neutrino mass matrix. Then, the Dirac neutrino mass matrix $h_{\nu}$ is converted 
to $U_{R}^{T} h_{\nu}$. However, for our convenience, we use $\bar{h}_{\nu}=U_{R}^{T} h_{\nu} U_{R}$ instead of $U_{R}^{T} h_{\nu}$ :

$$
\begin{aligned}
\bar{h}_{\nu} & =U_{R}^{T} h_{\nu} U_{R} \\
& \simeq h_{0}\left(\begin{array}{ccc}
1+\bar{\epsilon} & -\frac{\bar{\delta}_{1}}{2} & 2 \sqrt{2} i \bar{\epsilon}_{1} \sin \alpha_{1}+\sqrt{2} \bar{\epsilon} \\
-\frac{\bar{\delta}_{1}}{2} & 1-\bar{\epsilon}+\frac{\bar{\delta}_{1}}{2} & 0 \\
2 \sqrt{2} i \bar{\epsilon}_{1} \sin \alpha_{1}+\sqrt{2} \bar{\epsilon} & 0 & 1+\bar{\delta}_{2}
\end{array}\right) .
\end{aligned}
$$

\section{Numerical Study of Neutrino Mixings}

The lepton flavor mixing matrix $U[14]$ is obtained as

$$
U=F_{\ell}^{\dagger} F_{0}^{\dagger} V_{\nu}
$$

which gives

$$
\begin{aligned}
& \sin \theta_{12} \simeq-\frac{1}{\sqrt{2}}+\sqrt{\frac{m_{e}}{m_{\mu}}}+\frac{1}{\sqrt{2}} \frac{2|\epsilon| \cos \phi+\tilde{\epsilon}}{2 \delta_{1}}, \\
& \sin \theta_{13} \simeq \frac{2}{\sqrt{3}} \sqrt{\frac{m_{e}}{m_{\mu}}} \\
& \sin \theta_{23} \simeq-\frac{2}{\sqrt{3}}+\frac{1}{\sqrt{6}} \frac{m_{\mu}}{m_{\tau}}
\end{aligned}
$$

where $\theta_{i j}$ correspond to the mixing angles in the conventional parameterization of the mixing matrix in PDG [15]. Putting the experimental data with $3 \sigma$ in [16, 17],

$$
\begin{aligned}
& 7.2 \times 10^{-5} \mathrm{eV}^{2} \leq \Delta m_{12}^{2} \leq 9.1 \times 10^{-5} \mathrm{eV}^{2}, \quad 0.23 \leq \sin ^{2} \theta_{12} \leq 0.38 \\
& 1.4 \times 10^{-3} \mathrm{eV}^{2} \leq \Delta m_{13}^{2} \leq 3.3 \times 10^{-3} \mathrm{eV}^{2}, \quad \sin ^{2} 2 \theta_{23} \geq 0.90
\end{aligned}
$$

we obtain allowed regions of parameters in our model.

In the previous paper [2], we assumed that $\delta_{1}$ was much larger than $\epsilon_{i}(i=1,2,3)$ and $\epsilon$. Moreover, the only phase $\varphi$ was taken account for the $C P$ violation in the analyses. However, there is no reason to take such assumptions. Therefore, in this paper, parameters $\delta_{1}, \delta_{2}, \epsilon_{1}, \epsilon_{2}, \epsilon_{3}$ and $\epsilon$ are scanned in the region of $0-0.1$ while phases $\alpha_{1}, \alpha_{2}, \alpha_{3}, \beta_{1}, \beta_{2}$ and $\varphi$ are scanned in the region of $0-2 \pi$.

Fig. 2 shows the plot of allowed region on the $\delta_{1}-\epsilon_{i}(i=1,2,3)$ and $\delta_{1}-\epsilon$ planes. The 

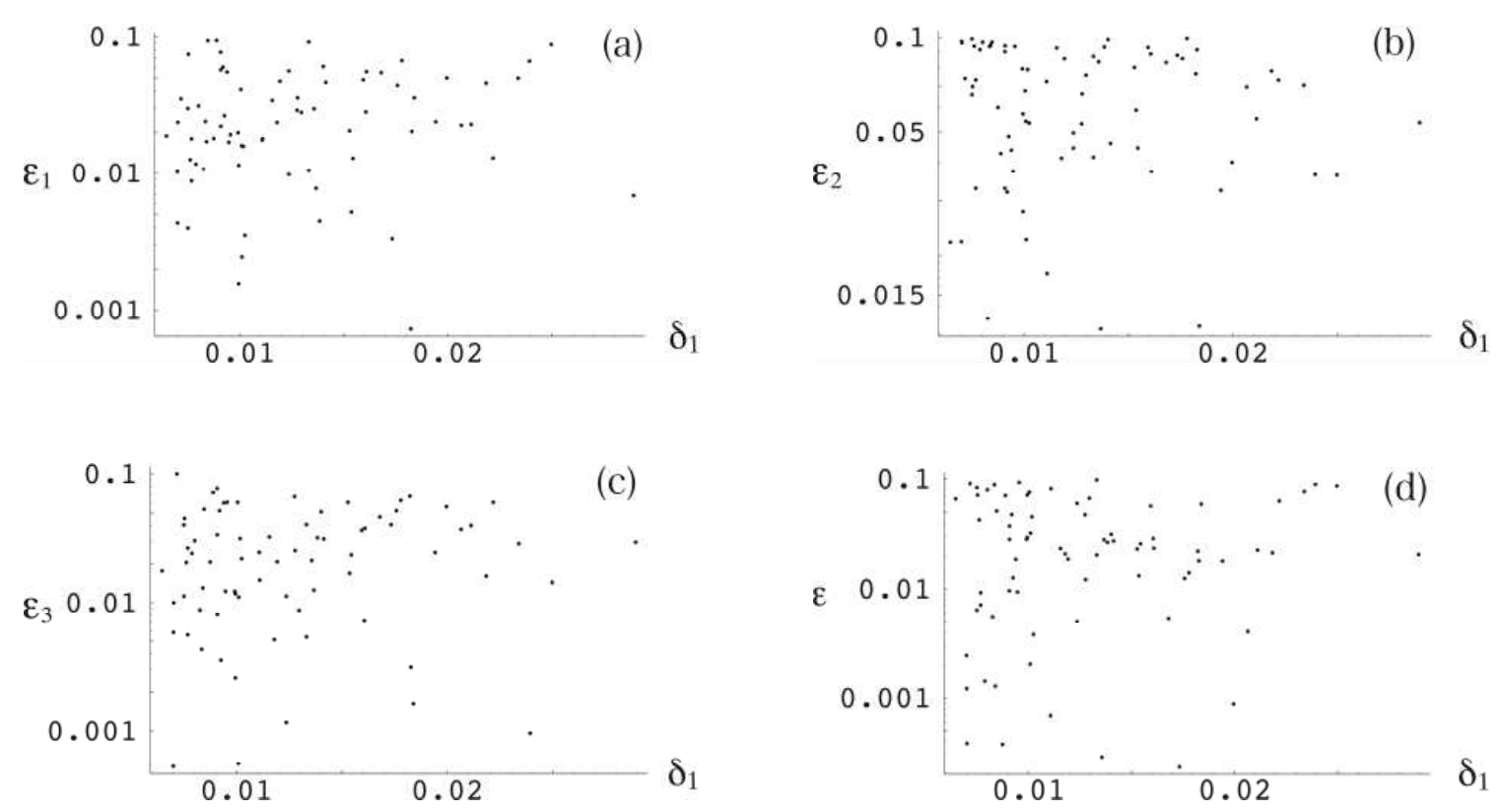

Figure 2: Allowed regions on the (a) $\delta_{1}-\epsilon_{1}$ plane, (b) $\delta_{1}-\epsilon_{2}$ plane, (c) $\delta_{1}-\epsilon_{3}$ plane and (d) $\delta_{1}-\epsilon$ plane.

magnitude of $\delta_{1}$ is allowed in 0.003-0.025 while $\epsilon_{1}, \epsilon_{3}$ and $\epsilon$ are allowed to be lower than 0.001, and $\epsilon_{2}$ is larger than 0.01 . We omit figures of allowed regions of phases since all phases are allowed in $0-2 \pi$.

Since the model leads to the inverted mass hierarchy for neutrinos, the lightest neutrino mass is $m_{3}$, which depends mainly on the parameter $\epsilon_{2}$. Fig. 3 shows the $\epsilon_{2}$ dependence of $m_{3}^{2}$. The predicted $m_{3}$ is (150) $\times 10^{-5} \mathrm{eV}$.

We can also discuss the neutrinoless double beta decay rate, which is determined by an effective Majorana mass:

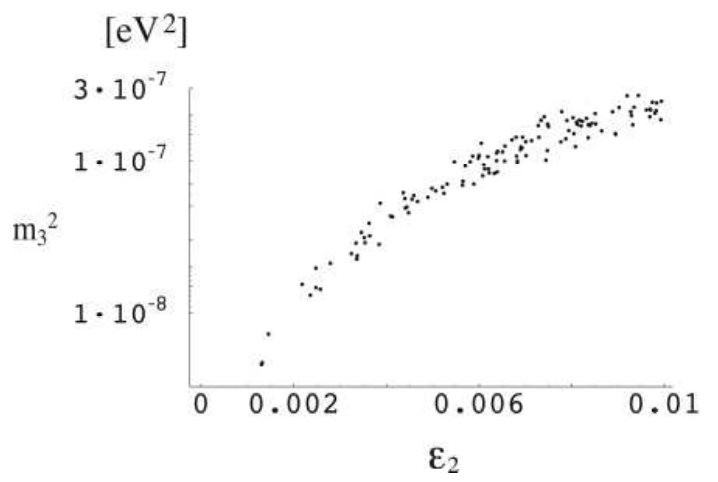

Figure 3: The square of the lightest neutrino mass, $m_{3}^{2}$ versus $\epsilon_{2}$.

$$
\langle m\rangle_{e e}=\left|m_{1} c_{12}^{2} c_{13}^{2} e^{i \rho}+m_{2} s_{12}^{2} c_{13}^{2} e^{i \sigma}+m_{3} s_{13}^{2} e^{-2 i \delta_{D}}\right|
$$

where $c_{i j}$ and $s_{i j}$ denote $\cos \theta_{i j}$ and $\sin \theta_{i j}$, respectively, $\delta_{D}$ is a so called the Dirac phase, 


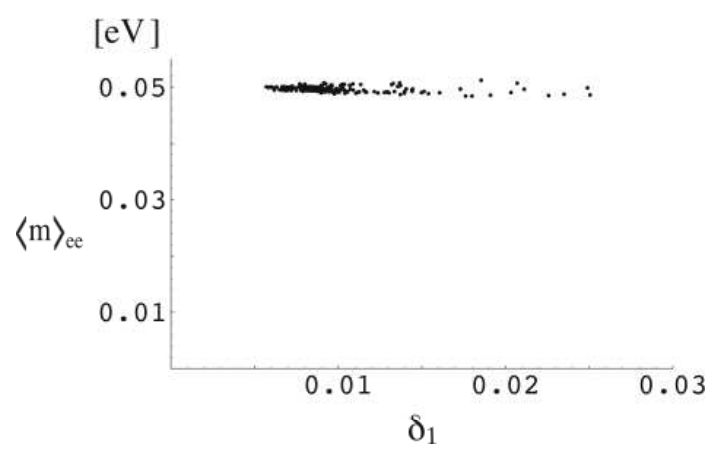

Figure 4: The predicted effective Majorana mass $\langle m\rangle_{e e}$ versus $\delta_{1}$.

and $\rho, \sigma$ are the Majorana phases. The Majorana phases are estimated from the mass matrix of Eq. (6). Therefore, the Majorana phases are at most of the order of $\epsilon_{i}(i=1,2,3)$ and $\epsilon$, which are very small. The predicted $\langle m\rangle_{e e}$ is presented in Fig. 4 , where $m_{1}=0.05$ $\mathrm{eV}$ is fixed. This numerical result is consistent with the one in the previous work [2], $\langle m\rangle_{e e} \simeq 50 \mathrm{meV}$, which is accessible to future experiments.

\section{Leptogenesis and $J_{C P}$}

In our scenario, the baryon asymmetry is explained by the leptogenesis [18, 19] via decays of the right-handed neutrinos $N_{i}$, which are produced non-thermally by decays of the inflaton $\varphi_{\text {inf }}$ [20].

Due to the non-vanishing phases in our model, CP invariance is violated in the Yukawa matrix $\bar{h}_{\nu}$. Then, the interference between decay amplitudes of tree and one-loop diagrams results in the lepton number production [18]. The lepton number asymmetry per decay of the right-handed neutrino $N_{i}$ into the left-handed lepton doublets $l_{L j}$ and the Higg scalar $H$ is given by [18, 21]

$$
\begin{aligned}
\epsilon_{\ell i} & \equiv \frac{\sum_{j} \Gamma\left(N_{i} \rightarrow l_{L j}+H\right)-\sum_{j} \Gamma\left(N_{i} \rightarrow \bar{l}_{L j}+\bar{H}\right)}{\sum_{j} \Gamma\left(N_{i} \rightarrow l_{L j}+H\right)+\sum_{j} \Gamma\left(N_{i} \rightarrow \bar{l}_{L j}+\bar{H}\right)} \\
& =-\frac{1}{8 \pi} \frac{1}{\left(\bar{h}_{\nu} \bar{h}_{\nu}^{\dagger}\right)_{i i}} \sum_{k \neq i} \operatorname{Im}\left[\left\{\left(\bar{h}_{\nu} \bar{h}_{\nu}^{\dagger}\right)_{i k}\right\}^{2}\right]\left[\mathcal{F}_{V}\left(\frac{m_{R k}^{2}}{m_{R i}^{2}}\right)+\mathcal{F}_{S}\left(\frac{m_{R k}^{2}}{m_{R i}^{2}}\right)\right],
\end{aligned}
$$

where $\mathcal{F}_{V}(x)$ and $\mathcal{F}_{S}(x)$ represent contributions from vertex and self-energy diagrams, 
respectively. In the case of the SUSY theory, they are given by [22]

$$
\mathcal{F}_{V}(x)=\sqrt{x} \ln \left(1+\frac{1}{x}\right), \quad \mathcal{F}_{S}(x)=\frac{2 \sqrt{x}}{x-1}
$$

Here, we assume that the mass difference of the right-handed neutrinos is large enough compared with their decay widths so that the perturbative calculation is ensured 1 .

The relevant elements of $h_{\nu} \bar{h}_{\nu}^{\dagger}$ are given as

$$
\begin{aligned}
& \operatorname{Im}\left[\left(\overline{\mathrm{h}}_{\nu} \overline{\mathrm{h}}_{\nu}^{\dagger}\right)_{12}{ }^{2}\right]=-\operatorname{Im}\left[\left(\overline{\mathrm{h}}_{\nu} \overline{\mathrm{h}}_{\nu}^{\dagger}\right)_{21}{ }^{2}\right] \simeq 2 \epsilon \delta_{1}^{2}\left(\cos \beta_{1}+\frac{\delta_{1}}{4}\right) \sin \left(\varphi-\beta_{1}\right), \\
& \left(\bar{h}_{\nu} \bar{h}_{\nu}^{\dagger}\right)_{11}=\left|h_{0}\right|^{2}\left(1+\delta_{1}-2|\epsilon| \cos \varphi\right), \\
& \left(\bar{h}_{\nu} \bar{h}_{\nu}^{\dagger}\right)_{22}=\left|h_{0}\right|^{2}\left(1+\delta_{1}+2|\epsilon| \cos \varphi\right) .
\end{aligned}
$$

Since the right-handed neutrino masses of the first and second families are almost degenerate in the present model, the self-energy contribution $\mathcal{F}_{S}(x)$ is much larger than the vertex contribution $\mathcal{F}_{V}(x)$ as follows:

$$
\begin{aligned}
& \mathcal{F}_{V}\left(\frac{m_{R 2}^{2}}{m_{R 1}^{2}}\right)=\mathcal{F}_{V}\left(\frac{m_{R 1}^{2}}{m_{R 2}^{2}}\right) \simeq \ln 2 \\
& \mathcal{F}_{S}\left(\frac{m_{R 2}^{2}}{m_{R 1}^{2}}\right)=-\mathcal{F}_{S}\left(\frac{m_{R 1}^{2}}{m_{R 2}^{2}}\right)=\frac{2 m_{R 1} m_{R 2}}{m_{R 2}^{2}-m_{R 1}^{2}} \simeq \frac{1}{2} \frac{\epsilon_{2}}{\epsilon_{1}^{2} \cos \left(2 \alpha_{1}-\alpha_{2}\right)-\epsilon_{3}^{2} \cos \left(\alpha_{2}-2 \alpha_{3}\right)} .
\end{aligned}
$$

Therefore, the magnitude of $\mathcal{F}_{S}\left(\frac{m_{R 2}^{2}}{m_{R 1}^{2}}\right)$ could be enhanced by the quasi-degenerate righthanded neutrinos. Then there occurs an enhancement of CP asymmetry for some region of the degeneracy. The scenario utilizing this enhancement is called as "resonant leptogenesis" [23, 24].

The lepton number asymmetry is given

$\epsilon_{\ell 1}=\epsilon_{\ell 2} \simeq-\frac{\left|h_{0}\right|^{2}}{8 \pi} \epsilon \delta_{1}^{2}\left(\cos \beta_{1}+\frac{\delta_{1}}{4}\right) \frac{\epsilon_{2}}{\epsilon_{1}^{2} \cos \left(2 \alpha_{1}-\alpha_{2}\right)-\epsilon_{3}^{2} \cos \left(\alpha_{2}-2 \alpha_{3}\right)} \sin \left(\varphi-\beta_{1}\right)$.

The value of $\left|h_{0}\right|^{2}$ is given in terms of $m_{1}$ and $m_{R 1}$ :

$$
\left|h_{0}\right|^{2} \simeq \frac{m_{1} m_{R 1}}{(174 \sin \beta \mathrm{GeV})^{2}}
$$

\footnotetext{
${ }^{1}$ In the quasi-degenerate case of the right-handed Majorana neutrinos, $\mathcal{F}_{S}(x)$ is modified as $\mathcal{F}_{S}(x)=$ $2(x-1) \sqrt{x} /\left\{(x-1)^{2}+\left(\Gamma_{k}^{0}\right)^{2} / m_{R i}^{2}\right\}$ with $\Gamma_{k}^{0} \simeq h_{0}^{2} m_{R k} /(8 \pi)$. As far as $x-1>h_{0}^{2}$, this assumption is guaranteed.
} 

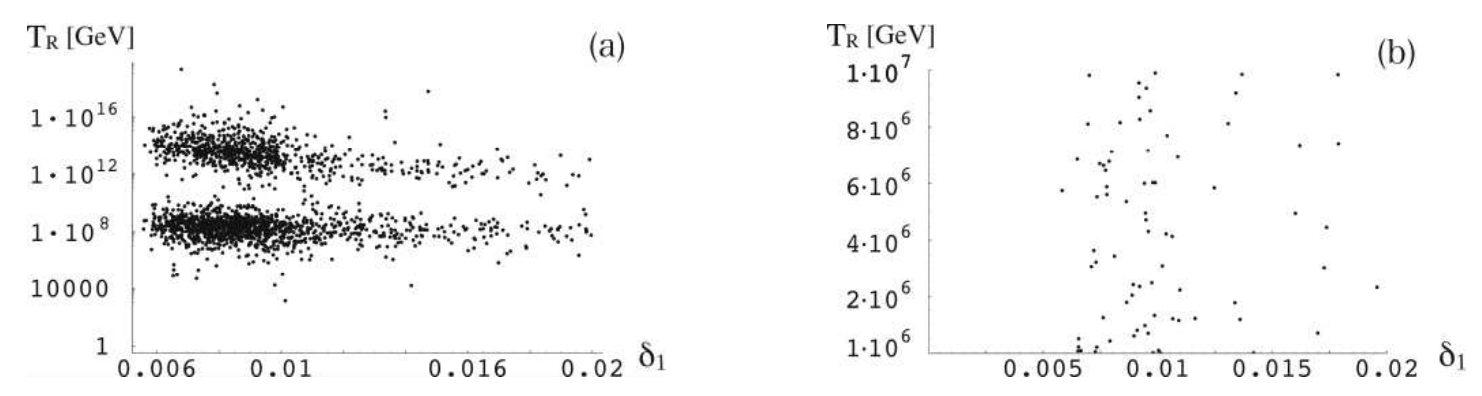

Figure 5: Allowed region of $T_{R}$ versus $\delta_{1}$ in order to explain the observed baryon asymmetry. The full region of $T_{R}$ is shown in (a), while the $10^{6}-10^{7} \mathrm{GeV}$ region of $T_{R}$ is shown in (b).

where the vacuum expectation value of the Higgs $H_{u}$ is taken as $\left\langle H_{u}\right\rangle=174 \sin \beta \mathrm{GeV}$ $\left(\beta=\tan ^{-1}\left\langle H_{u}\right\rangle /\left\langle H_{d}\right\rangle\right)$. The ratio of the lepton number density $n_{L}$ to the entropy density $s$ produced by the inflaton decay is given by [20]

$$
\frac{n_{L}}{s}=\frac{3}{2} \sum_{i} \epsilon_{\ell i} B_{r}^{(i)} \frac{T_{R}}{m_{\phi_{i n f}}},
$$

where $T_{R}$ is the reheating temperature after the inflation, $m_{\phi_{i n f}}$ is the mass of the inflaton, and $B_{r}^{(i)}$ is the branching ratio of the decay channel of the inflaton to $N_{i}$, i.e., $B_{r}^{(i)}=$ $B_{r}\left(\phi \rightarrow N_{i} N_{i}\right)$. Here, we have assumed that the inflaton decays into a pair of righthanded neutrinos, and $m_{R i}>T_{R}$ in order to make the generated lepton asymmetry not washed out by lepton-number violating processes after the $N_{i}$ 's decay. The dominant term in the right hand side of Eq. (36) follows from $i=1$ and 2 because $m_{R 3}$ is much heavier than $m_{R 1}$ and $m_{R 2}$. A part of the produced lepton asymmetry is immediately converted [18] into the baryon asymmetry via the "sphaleron" effect [25] since the decays of $N_{i}$ take place much before the electroweak phase transition. The baryon asymmetry is given by

$$
\frac{n_{B}}{s}=C \frac{n_{L}}{s}
$$

where $C$ is given by $C \simeq-0.35$ in the minimal SUSY standard model [26]. Therefore, $\epsilon_{\ell 1}$ should be negative in order to explain the sign of $n_{B} / s$. 
Now, we can obtain the allowed region of $T_{R}$ to reproduce the amount of the observed baryon asymmetry $n_{B} / s \simeq(0.8-0.9) \times 10^{-10}$ [15] in our model. Fig. 5(a) presents the allowed region of $T_{R}$ versus $\delta_{1}$, where $2 m_{R 1(2)} / m_{\phi_{i n f}} \simeq 1$ and $B_{r}^{(1)}+B_{r}^{(2)} \simeq 1$. The leptogenesis works even in the region of $T_{R}=10^{4}-10^{6} \mathrm{GeV}$, which is lower than the expected value, $10^{7} \mathrm{GeV}$, in the previous paper [2] because of the enhancement, so called the resonant leptogenesis. We also show the region of $T_{R}=10^{6}-10^{7} \mathrm{GeV}$ in Fig. 5(b).

There is another $C P$ violation, which is the low energy phenomena and is expected to be measured in the future terrestrial experiments. It is very interesting to ask whether there

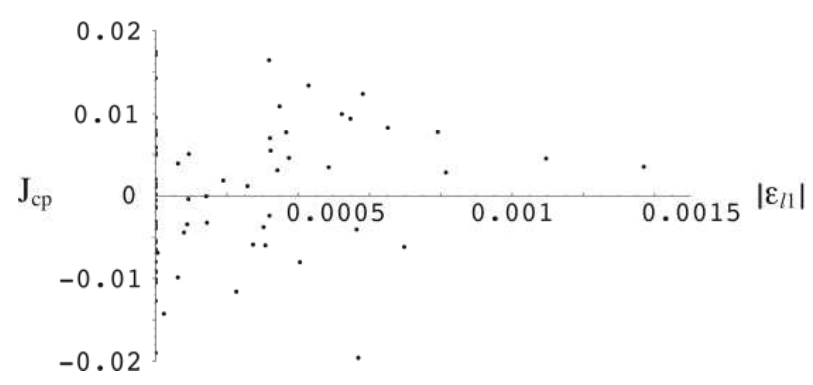
is the correlation between observed baryon asymmetry and to be negative in order to give positive $n_{B} / s$. the leptonic $C P$ violation at the low energy. In order to answer this question, we plot allowed region on the plane of $\epsilon_{\ell 1}$ and $J_{C P}$, which is the Jarlskog invariant [27] in Fig. 6. We cannot find the correlation between them as seen in Fig. 6. Both positive and negative $J_{C P}$ are allowed for the negative $\epsilon_{\ell 1}$. The $\epsilon_{\ell 1}$ is given mainly in terms of phases $\beta_{1}$ and $\varphi$ as seen Eq. (34) while the magnitude of $J_{C P}$ is determined mainly by all phases except for $\varphi$. For example, $\left|\epsilon_{\ell 1}\right|$ reaches 0.001 but $J_{C P}$ is at most $4 \times 10^{-6}$ if $\varphi$ is the only non-vanishing phase. On the other hand, $\left|\epsilon_{\ell 1}\right|$ is at most $10^{-8}$ but $J_{C P}$ could be 0.01 in the case of non-vanishing $\alpha_{1}$, $\alpha_{2}, \alpha_{3}$ and $\beta_{2}$ with $\varphi=0$ and $\beta_{1}=0$. Only $\beta_{1}$ can contribute considerably both $\epsilon_{\ell 1}$ and $J_{C P}$. 


\section{Summary}

We have presented the comprehensive analyses of the model with inverted hierarchy of the neutrino masses, which is based on the $\mathbf{T}^{\mathbf{2}} / \mathbf{Z}_{\mathbf{3}}$ orbifold model [3]. Here, a $\mathbf{5}^{*}$ and a right-handed neutrino $N$ in each family are localized on each of the equivalent three fixed points of the $\mathbf{T}^{\mathbf{2}} / \mathbf{Z}_{\mathbf{3}}$ orbifold while three 10's and Higgs doublets $H_{u}$ and $H_{d}$ live in the bulk. The Higgs field $\phi$ responsible for the B-L breaking is assumed to be localized on the fixed point of the third family of $5^{*}$ and $N$.

While our analyses justify the qualitative result in the previous paper [2], new results are also obtained. The $m_{3}$ is predicted in the region $(1-50) \times 10^{-5} \mathrm{eV}$. The model also predicts the element of the neutrino mass matrix, $\langle m\rangle_{e e}$, responsible for neutrinoless double beta decays as $\langle m\rangle_{e e} \simeq 50 \mathrm{meV}$, which has already been predicted in the previous paper [2]. We have shown that the observed baryon asymmetry in the present universe is produced by the non-thermal leptogenesis via the inflaton decay. Due to the quasi-degenerate right-handed Majorana neutrino, the baryon asymmetry is enhanced. In conclusion, we have found that the leptogenesis works even at the reheating temperature $T_{R}=10^{4}-10^{6} \mathrm{GeV}$. The low energy $C P$ violation $J_{C P}$ could be 0.02 , however there is no correlation between $\epsilon_{\ell 1}$ and $J_{C P}$.

\section{Acknowledgement :}

We thank T. Yanagida for suggesting numerical studies of the model. We also thank H. Sawanaka for his help. The work of M.T. has been supported by the Grant-in-Aid for Science Research of the Ministry of Education, Science, and Culture of Japan No. 17540243.

\section{References}

[1] T. Kobayashi, H.P. Nilles, F. Plöger, S. Raby and M. Ratz, hep-ph/0611020. 
[2] M. Tanimoto and T. Yanagida, Phys. Lett. B633 (2006) 567.

[3] T. Watari and T. Yanagida, Phys. Lett. B544 (2002) 167.

[4] T. Watari and T. Yanagida, Phys. Rev. D70 (2004) 036009.

[5] M. Fukugita, M. Tanimoto and T. Yanagida, Phys. Rev. D57 (1998) 4429;

M. Tanimoto, Phys. Rev. D59 (1999) 017304.

[6] H. Fritzsch and Z. Xing, Phys. Lett. B372 (1996) 265; ibid. B440 (1998) 313.

[7] N. Arkani-Hamed and S. Dimopoulos, Phys. Rev. D65 (2002) 052003.

[8] T. Yanagida, in Proceedings of the "Workshop on the Unified Theory and the Baryon Number in the Universe", Tsukuba, Japan, 1979, edited by O. Sawada and A. Sugamoto, KEK Report No. KEK-79-18, p. 95; Prog. Theor. Phys. 64 (1980) 1103;

P. Ramond, in Talk given at the Sanibel Symposium, Palm Coast, Fla., 1979, preprint CALT-68-709;

S.L. Glashow, in Quarks and leptons, proceedings of the advanced study institute (Cargèse, Corsica, 1979), J.-L. Basdevant et al. eds., Plenum, New York 1981.

[9] For an early work, see P. Minkowski, Phys. Lett. B67 (1977) 421.

See also R.N. Mohapatra and G. Senjanovic, Phys. Rev. Lett. 44 (1980) 912.

[10] Super-Kamiokande Collaboration (S. Fukuda et al.), Phys. Rev. Lett. 86 (2001) 5651, 5656

SNO Collaboration (Q. R. Ahmad et al.), Phys. Rev. Lett. 87 (2001) 071301; ibid. 89 (2002) 011301; ibid. 89 (2002) 011302; ibid. 92 (2004) 181301.

[11] KamLAND Collaboration (K. Eguchi et al.), Phys. Rev. Lett. 90 (2003) 0212021; KamLAND Collaboration (T. Araki et al.), Phys. Rev. Lett. 94 (2005) 081801. 
[12] Super-Kamiokande Collaboration (Y. Fukuda et al.), Phys. Rev. Lett. 81 (1998) 1562; ibid. 82 (1999) 2644; ibid. 82 (1999) 5194.

[13] Y. Koide, Phys. Rev. D28 (1983) 252, Phys. Rev. D39 (1989) 1391.

[14] Z. Maki, M. Nakagawa and S. Sakata, Prog. Theor. Phys. 28 (1962) 870.

[15] S. Eidelman, et al. [Particle Data Group Collaboration], Phys. Lett. B592 (2004) 1.

[16] M. Maltoni, T. Schwetz, M. Tortola and J.W.F. Valle, New J. Phys 6 (2004) 122.

[17] G.L. Fogli, E. Lisi, A. Marrone and A. Palazzo, Prog. Part. Nucl. Phys. 57 (2006) 742 .

[18] M. Fukugita and T. Yanagida, Phys. Lett. B174 (1986) 45.

[19] For reviews and references, see, for example, W. Buchmuller and M. Plumacher, Int. J. Mod. Phys. A15 (2000) 5047;

W. Buchmuller, R.D. Peccei and T. Yanagida, Ann. Rev. Nucl. Part. Sci. 55 (2005) 311.

[20] K. Kumekawa, T. Moroi and T. Yanagida, Prog. Theor. Phys. 92 (1994) 437;

T. Asaka, K. Hamaguchi, M. Kawasaki and T. Yanagida, Phys. Lett. B464 (1999) 12; Phys. Rev. D61 (2000) 083512;

G. F. Giudice, M. Peloso, A. Riotto and I. Tkachev, JHEP 9908 (1999) 014.

See also G. Lazarides and Q. Shafi, Phys. Lett. B258 (1991) 305.

[21] M. Flanz, E. A. Paschos and U. Sarkar, Phys. Lett. B345 (1995) 248 [Erratum-ibid. B384 (1995) 487];

L. Covi, E. Roulet and F. Vissani, Phys. Lett. B384 (1996) 169;

W. Buchmuller and M. Plumacher, Phys. Lett. B431 (1998) 354.

[22] L. Covi, E. Roulet and F. Vissani, in Ref. 21]. 
[23] A. Pilaftsis, Phys. Rev. D56 (1997) 5431; Int. J. Mod. Phys. A 14 (1999) 1811.

[24] A. Pilaftsis and Thomas E.J. Underwood, Nucl. Phys. B693 (2004) 303.

[25] V. A. Kuzmin, V.A. Rubakov and M. E. Shaposhnikov, Phys. Lett. B155 (1985) 36.

[26] S. Y. Khlebnikov and M. E. Shaposhnikov, Nucl. Phys. B308 (1988) 885;

J. A. Harvey and M. S. Turner, Phys. Rev. D42 (1990) 3344.

[27] C. Jarlskog, Phys. Rev. Lett. 55 (1985) 1039. 